\title{
PERCEPCIÓN DEL CONOCIMIENTO DE LAS EXPRESIONES CULTURALES Y SU REVALORIZACIÓN INTEGRAL EN LA COMUNIDAD ALTOANDINA, CARAZ, ANCASH, PERÚ
}

\author{
Mildred Paredes T. ${ }^{1}$ Héctor Hernández V., Luís Vicuña P., Vladimir Arias A., José Rivera B. \\ Universidad Nacional Mayor de San Marcos, Lima, Perú \\ (RECIBIDO EL 2/11/2009, ACEPTADO EL 2/12/ 2009)
}

\begin{abstract}
RESUMEN
Las expresiones culturales pertenecientes a un ámbito específico expresan la diversidad de identidades culturales al vincular contenidos, valores y modos de vida UNESCO (2005); al desarrollarse en bienes y servicios posibilitarían a las comunidades campesinas andinas peruanas su inclusión socioeconómica mediante estos productos para un mercado potencial no existente aún en la zona de estudio.

Se pregunta ¿cuál es el efecto de la aplicación del taller sobre el valor agregado de los productos culturales para su comercialización en pobladores de los caseríos de la comunidad Cruz de Mayo de Caraz, Ancash?, en tanto los pobladores de dos caseríos desconocen que estos productos podrían tener valor económico para la comunidad campesina Cruz de Mayo del Callejón de Huaylas, Caraz, Ancash. Investigación aplicada; diseño cuasiexperimental; previamente se exploró y registró los factibles productos de expresiones culturales, aplicándose la Escala de Percepción de 20 ítems con cuatro grados de valoración; confiabilidad: 0,90 y validez de contenido por criterio de jueces y capacidad de discriminación; análisis de varianza de un factor y para comparar el t student para grupos independientes.
\end{abstract}

Objetivos: 1) Identificar la medida en que los talleres sobre valor agregado afectan la percepción de los productos culturales para su comercialización en los pobladores adultos de los caseríos de la comunidad Cruz de Mayo de Caraz, Ancash.

Hipótesis: El taller sobre valor agregado afecta significativamente la percepción de los productos culturales para su comercialización en pobladores de los caseríos de la Comunidad Campesina Cruz de Mayo de Caraz, Ancash.

Resultado: Se rechaza la hipótesis nula Ho; ya que hubo disminución significativa en la percepción de la aplicación de valor agregado a los productos culturales. $(\mathrm{t}=-2.19$ al $0,05)$.

Palabras clave: Percepción, percepción a productos culturales, percepción del valor agregado, bienes culturales, servicios culturales, diversidad cultural.

1 Docente Asociada , Facultad de Psicología UNMSM E-mail: mildredparedestarazona@gmail.com 


\section{ABSTRACT \\ PERCEPTION CULTURAL EXPRESSIONS OF KNOWLEDGE AND INTEGRATED PROMOTION IN THE ANDEAN COMMUNITY, CARAZ ANCASH, PERU}

Cultural expressions belonging to a particular subject area reflect the diversity of cultural identities by linking content, values and lifestyles (UNESCO, 2005), to develop goods and services would enable socio-economic inclusion of the Peruvian Andean peasant communities with products for a market potential is still existing in the study area.

Problem: Which is the effect of the application of the workshop on the added value of the cultural products in inhabitants of small villages of the Comunidad Campesina Cruz de Mayo of Caraz, Ancash? In the meantime the inhabitants of two groups of houses are ignorant of that these products would be able to have value aggregation for the Peasant Community Cruz de Mayo of Callejón de Huaylas, Caraz, Ancash.

Applied research, cuasiexperimental design; Previously scanned and posted the feasible products of cultural expressions, applying over oneself Perception's ladder of 20 items with four grades of assessment; Reliability: 0.90 and validity of contents for opinion of judges and capability of discrimination.

Hypothesis: The workshop on added value found the perception of the cultural products for his commercialization in residents of the community Cruz de Mayo, Ancash.

Result: H1, since sensible decrease in the perception of the application of added value had the cultural products accepts the alternating hypothesis himself. ( $t=-2,19$ to the 0.05 ).

Keywords: Perception, perception to cultural products, perception of the ad valorem, cultural goods, cultural services, cultural diversity.

\section{INTRODUCCIÓN}

Se hace referencia como cultura de la comunidad campesina andina a un conjunto de elementos que le son afines a una población ubicada en un universo social y geográfico complejo; con la evidencia de la diversidad local, regional y étnica manifiesta en costumbres, rituales y valores inscritos en la tradición de cada pueblo tanto de la costa, como de la sierra o la selva. Al ser la comunidad una unidad social de larga data, si bien no mantiene sus raíces milenarias, su autenticidad y su originalidad plena; sin embargo, esta unidad social es la más antigua y la más democrática que la justicia peruana tiene en su seno (Kapsoli, E., W., 1989, 16).

De ahí Adams y Valdivia (1994) refieren que es posible ubicar una serie de rasgos culturales comunes que responden a una organización social sustentada en particulares condiciones de reproducción en los andes, siendo un patrón su diversidad cultural, tanto para cada caserío como integralmente para la comunidad campesina andina. Incuestionablente hay potencial a desarrollarse en recurso intangible y tangible, riqueza a ser expresada en bienes y servicios de sus expresiones culturales. Actualmente, los productos tienen un bajo o escaso valor agregado, lo que va postergando su oportunidad de crecimiento en una óptica de desarrollo de producto comercial; sin desmerecer su valor intríseco, estos productos requieren un proceso de calidad y cumplir ciertos estándares que demanda el consumidor, mercado potencial en vías a constituirse en un sector económico que les posibilite una inclusión socioeconómica con índices de calidad de vida. 
En la comunidad de Cruz de Mayo, en sus caseríos de Queral, Pampacocha, Chinchay y Miraramar, Caraz, Ancash, se observa que sus miembros manifiestan aparentemente el descrédito, a que estos productos se constituyan como sector económico, de ahí se hizo la pregunta ¿Cuál es el efecto de la aplicación del taller sobre el valor agregado de los productos culturales para su comercialización en jóvenes y adultos de los caseríos de la comunidad Cruz de Mayo? con el objetivo: 1. Identificar las expresiones culturales que puedan desarrollarse en bienes y servicios culturales comerciables de la Comunidad Campesina de Cruz de Mayo; 2 . Identificar en qué medida los talleres sobre valor agregado afectan la percepción de los productos culturales para su comercialización en los pobladores adultos de los caseríos de la comunidad Cruz de Mayo.

Con el propósito de explicar la percepción de las expresiones culturales con la percepción de valor agregado de los productos culturales se reunirá información que sustente las estrategías de gestión de las comunidades campesinas alto-andinas según la demanda de productos y bienes culturales en productos complementarios que coadyuven a su ingreso familiar, en una primera etapa a mediano plazo, orientado a propiciar y favorecer su inclusión económica a la región y al ámbito nacional.

Se evidencia que, el conocimiento de la oferta de demanda es el factor de sinergia para las actividades económicas con beneficio pecuniario mediante articulaciones empresariales entre los miembros de los Caseríos mencionados (Paredes, T. Mildred; Hernandez V. Héctor; Vicuña P. Luis; De Miguel, C. Jesús Ma., 2007), posibilita de que los bienes y servicios culturales se constituyan en un producto ofertable, tal como lo señala la UNESCO (2005) para la comunidad, por cuanto estas expresiones en productos culturales pertenencen a un ámbito específico que los ubica más alla de sus aspectos puramente comerciales, al vincular contenidos, valores y modos de vida que expresan la diversidad de identidades culturales; que de lograr satisfacer a los consumidores de estos productos, formarían su mercado al ser identificados por su originalidad, así mismo obtendrían un mayor beneficio económico como artífices y poseedores del gran acervo cultural.

En tal sentido, cobra realce conocer aquellas variables psicológicas para predecir el comportamiento e intervenir de manera efectiva disponiendo de información de aquella dimensión subjetiva mediante la percepción, variable psicosocial que cumple el cometido de interpretar la realidad por sus artífices en lo cognitivo, proporcionando indicadores objetivos para aproximarse como "es propio asumir y aceptar que dichas expresiones poseen un transfondo económico y beneficios para sus actores" (Uribe, P. UNESCO, 2005), en concordancia a la naturaleza de la organización comunitaria en el marco de "un deber percibir al Perú desde una perspectiva pluricultural, es decir no homogénea, lo que posibilitaría un acercamiento distinto a las múltiples manifestaciones de la realidad peruana, que nos obliga a una definición del Perú como plurinacional" (Solis, G., 2006, 9).

El estudio de la percepción económica al valor agregado de los participantes provenientes de los sectores deprimidos y excluidos de nuestro país es pertinente a la universidad y concordante con sus políticas de investigación señaladas por el Vicerrectorado de Investigación. De igual manera, no es ajeno a la comunidad científica y profesional tener en cuenta las interrelaciones entre las actitudes y percepciones con el comportamiento económico (Katona, G., 1965). Quintanilla (2003) señala que la obra de Katona puso al 
descubierto la gran importancia de las expectativas, entendidas por este autor como una subclase de actitudes que se proyectan hacia el futuro. En consecuencia, las perspectivas en el tiempo se producen en los consumidores tanto hacia atrás como hacia delante de manera altamente selectiva. Por otra parte, las respuestas ante un mismo estímulo económico varían grandemente entre sujetos, así como en una misma persona en dos puntos diferentes del tiempo.

De este modo, los procesos psicológicos constituidos en categorías favorables hacia la conducta emitida por la cercanía al objeto medible como son las expresiones culturales en objetos comerciables, se incorporan, a nivel actitudinal al constituirse un patrón o norma de conducta económica, porque se requiere el hecho fáctico de la vivencia o la exposición al estímulo y la práctica al mismo para la emisión de una nueva conducta (González, Raúl, 1989: 13-31), y el aprendizaje forma la percepción de todo agente económico en una cognición socialmente compartida, en este caso, la membresía del caserío, la que impondrá a sus integrantes como norma de comportamiento ya que tales presiones serán más fuertes en ambientes más naturales y en fuerte relación a temas de mayor significación para los participantes. Cartwright, D. y Zander, A. (1985: 159)

En el aprendizaje de la actitud, a nivel cognitivo formula y mide directamente percepciones, pensamientos y factores evaluativos que son derivados, según su importancia relativa, a partir de estados de necesidad, y estos procesos preceptúales y ciertas actitudes significativas desarrolladas por los individuos para explorar el mundo que los rodea, permite explicar sus comportamientos económicos, concluye Albou en Descouvières, C. (1998), en tanto todo estímulo contextualizado, en la cultura del Caserío, tendrá significancia en la percepción del agente económico como es una unidad productiva.

La diversidad de expresiones culturales que asombran por su belleza y creatividad en zonas rurales del Perú no es desconocida para propios y extraños; a la fecha el pronóstico de sostenibilidad y de dirección por una gestión de calidad, se vincula a un alto porcentaje de información, lo que, hoy por hoy, es el conocimiento requerido y recurso de factibilidad para un proyecto económico sustentable. Los planes y programas proyectados u orientados a lograr la ansiada competitividad en la zona de estudio se presentan con resultados poco alentadores y alejados de las expectativas de la población demandante, peor aún, no atinan a cómo o en qué hacerlo, y lo que es real, parten de las opciones de una cultura para imponer a otra por estar ausente el conocimiento de los niveles de decisión la información pertinente; tal es el caso de la psicológica, así por ejemplo, si se tiene en consideración que el concepto de acomodación y consecuentemente de adaptación del esquema piagetano que vincula lo biológico y cultural, en consecuencia es el problema de percepción y cultura, como enfatiza Rivera B.,J.C. Ello complejiza superar la defectuosa y confusa información que se ha venido transmitiendo y que todavía se mantiene en el imaginario popular para captar, interpretar y aplicar el pensamiento del poblador andino, (Paredes, M y González del Río G. C., 2000: 106)

La cultura y el medio físico ejercen una influencia dominante en el comportamiento y manejo económico; forma de vida que el hombre adopta de modo creativo o habitual en cada caserío de la comunidad, sujeto a las potencialidades que ofrecen el medio físico y especialmente la riqueza de la diversidad cultural de los pueblos andinos peruanos, identificados como 
expresiones culturales. Estas potencialidades son los elementos básicos para superar carencias que no están apaleadas con los productos que les sirven de sobrevivencia para proveerse y amortiguar sus necesidades básicas de alimentación, salud, educación, vivienda, vestimenta, entre las prioritarias; necesidades evaluadas y percibidas por debajo del índice de calidad de vida que el medio económico ofrece; por ejemplo en el caserío de Queral la percepción es de mayor pobreza que los demás; y Papampacocha entre los que tiene una mejor percepción a sus condiciones de trabajo. Para elaborar sus viviendas los pobladores de esta zona, aprovechan de la naturaleza los recursos naturales como: la tierra para hacer sus adobes y los troncos de eucaliptos para construir sus casas y techarlas con tejas en mayor porcentaje, lo que es muy saludable para el paisaje y la salud. Según la topografía del terreno, las familias se han ubicado en lugares aparentemente de menor riesgo; mas no por ello se recalca que en sus terrenos agrícolas se observa deslizamientos (en Paredes T. M.; De Miguel, C Jma; Hernández V.H.; y Vicuña P. L., 2005)

Otro aspecto preocupante que se visualiza en la zona es que el empleo agrícola, considerando a la población de 15 años a más, ha bajado de $46 \%$ en 1993 a 15\% en el 2005 (INE, 2005), y que el empleo minero no es un sector clave dinamizador económico de la zona, es necesario encontrar nuevas formas de incrementar el empleo y la productividad en sectores específicos de la agricultura, al igual que explorar formas potenciales en otras actividades; teniendo una caracterización de diversificación de portafolio lo que daría poder de sostenibilidad para iniciar y mantener el desarrollo de la zona; por ello es necesario investigar cuál es la nueva recomposición de la estructura del empleo actual, se hace necesario, a fin de posibilitar un empleo de calidad, por ejemplo, con el desarrollo de productos de expresiones culturales en bienes y servicios para el mercado exterior y para el turismo, que le sirva a la población como un portafolio defensivo para generar ingresos alternativos cuando las condiciones agrícolas les sean adversas.

La competencia de la psicología en la prospectiva multidisciplinaria es coadyuvar al desarrollo sostenible, mediante la instrumentación de modelos de educación en programas de socialización económica vinculados a la capacitación laboral de manera específica, en la identificación y fortalecimiento de competencias individuales y organizacionales, construidas con productos económicos que favorezcan el desarrollo humano y a la preservación del medio ambiente ecológico.

\section{Referencias teóricas}

La Convención sobre la protección de la diversidad de las expresiones culturales del 20 de octubre de 2005 (UNESCO, 2005) es una respuesta a la necesidad imperiosa de las naciones y pueblos que se ven desprotegidos ante la complejidad y el juego de intereses entre agentes económicos y la desigualdad en el intercambio comercial, trato no equitativo por prácticas mercantilistas que desvirtúan el desarrollo sustentable de las minorías. El Perú por su "diversidad cultural es una rica virtualidad de potencialidades únicas para enfrentar los infinitos y sorprendentes retos que contrapone al hombre su realidad geográfica social" Solís, G. (2006: 23) y entendiendo que la expresión cultural "reposa en el principio de que los bienes y servicios culturales por su íntima relación con la cultura pertenecen a un ámbito específico que los ubica más allá de sus aspectos puramente comerciales: en 
efecto, ellos vinculan contenidos, valores, modos de vida que expresan las identidades culturales de una comunidad y reflejan la diversidad creadora de sus individuos" (Uribe, P., 2005).

Así mismo, se considera a las expresiones culturales como un potencial que puede considerarse como un producto cultural para concretar cadenas productivas, como también productos complementarios a los productos estacionarios de la zona, al no disponerse la estructuración vertical geográfica que les proporcionaba recursos diversos para su intercambio o consumo y posibilitar la recomposición de la estructura de empleo actual.

En la medida que estas expresiones culturares son productos, entendiendose por Bienes culturales a “... aquellos bienes de consumo que vinculan ideas, valores simbólicos y modos de vida y a influir sobre prácticas culturales; contribuyen con una oferta rica y diversa"; y por Servicios culturales ... aquellas actividades que, sin tomar la forma de un bien material, responden a una idea o a una necesidad de orden cultural y se traducen en medidas de apoyo a prácticas culturales" (UNESCO, 2004) que por su naturaleza tienen impacto faborable al medio ambiente y en su proceso no requieren materiales contaminantes ni reactivos que pudieran alterar el ecosistema.

Este potencial cultural, mayormente en las zonas alto andinas, se desvirtúa al observarse que aún no lleva a sus actores al logro económico, especialmente al tener la virtualidad de potencialidades para los productos de las expresiones culturales, así mismo por los reportes de los resultados de las investigaciones realizadas con la población de los Caseríos Pampacocha, Queral, Chinchay y Miramar pertenecientes a la Comunidad Campesina Cruz de Mayo, Callejón de Huaylas, Caraz; se concluye:

1. La construcción social de la organización de la comunidad campesina andina es, sui generis y portadora de calidad "psicológica"; que es propia y se diferencia por caseríos en su representación social; organización tipificada, entre otras dimensiones psicosociales, primeramente productividad e identidad, para las escalas tanto actitudinal e imagen; dimensiones fuertemente arraigadas en sus integrantes que permite señalar la existencia de un alto indice de identidad (Paredes T., Mildred \& De Miguel C. Jesús Ma., 2004);

2. El Caserío discrimina la percepción hacia las condiciones de trabajo; y la percepción al Manejo del medio ambiente es atribuida a la Comunidad. El Caserío de Pampacocha posee una mejor percepción de sus condiciones de trabajo difiriendo al de Queral (Paredes T. M. y colaboradores, 2005: 57-67).

3. La percepción del conocimiento de la demanda por la oferta demandada es el factor significativo para formar articulaciones organizacionales entre los miembros de cada caserio de la comunidad (Paredes T., M. y Colaboradores (2007-109).

Los trabajos pioneros en los pobladores Comunidad Campesina de Lacabamba- Prov. Pallasca, Ancash, Perú (IIGEO-UNMSM 2004, 2005) y los lineamientos del Plan Desarrollo de la Comunidad Campesina Espíritu Santo de Chacayan, Pasco 2006-2016 UNMSM/CENTROMIN PERU. IGEO (2006) precisan los niveles de pobreza y líneas estratégicas para revertirlas de acuerdo a la población estudiada; condición por la que atraviesa la Zona objetivo del presente estudio. 


\section{Procesos perceptivos y cultura}

La percepción se constituye un estilo cognitivo (Witkin, H. y Goodenough, D., 1981: 11-12), como tal, es un constructo que explica además la "forma en la evaluación de la conducta de los demás y de los propios estados o expectativas en un contexto, así como el reconocimiento de las emociones, en la decisión; y forma impresiones y procesos de atribución". Siendo un proceso psicológico demanda etapas de actividad psicológica que describe Santoro, E. (1980: 77-108): percibir no es recibir pasivamente el estímulo: es seleccionar, formular hipótesis, decidir y procesar la estimulación, eliminando, aumentando o disminuyendo aspectos de ella. Esta evaluación se inica en la percepción, proceso que tiene como competencia la categorización y significación construida o formada por el pensamiento, que se va formando y logrando su nivel superior mediante el aprendizaje en la cultura.

Cultura se refiere principalmente al fundamento o marco de referencia para una conducta aprendida, como algo distinto a lo que viene dado por la naturaleza o la biología, otorgando así al término de la cultura a ser utilizado para designar todo lo que se ha producido humanamente, tangible o intangible; por ejemplo hábitos, creencias, arte y artefactos, así como a los modos de trabajar; y que dicha producción ha pasado de una generación a otra, más que como un conjunto de tradiciones, reglas, símbolos que toman forma y se representan como sentimientos, pensamientos y conductas de grupos de personas, sino también como espíritu. En esta formulación, la cultura se distingue de la naturaleza y diferencia a una sociedad de otra.

De ahí, se asume que cada cultura "tiene una típica percepción de la realidad, la que surge de la simbolización y categorización de los elementos del entorno de la sociedad y que es trasmitida implícita y explícita en el lenguaje y expresada en el comportamiento" (González del Río, C. y Paredes T., M, 1999: 125).

Para enfocar el desarrollo de los conceptos económicos, se ha de considerar el aporte de Piaget, un teórico del desarrollo intelectual que adoptó un enfoque estructuralista genético; para él, la interacción entre los orígenes ontogenéticos de la infancia en lo individual y el medio ambiente crea estructuras lógicas sucesivas que regulan el proceso cognitivo. Considera que las características de estas estructuras y su orden de aparición son de aplicación universal, resultados de procesos adaptativos entre organismos humanos -cuya herencia biológica es la misma en todo el mundo- y medios ambientes con propiedades físicas fundamentales (coordenadas de espacio y tiempo, comportamiento de los objetos bajo la acción de fuerzas gravitacionales, etc.).

Las investigaciones sobre la inteligencia sensomotriz ponen de manifiesto la universalidad de estas estructuras intelectuales y su orden de aparición. El papel que Piaget asigna a la cultura es el de acelerar el proceso de desarrollo, orientar en términos de adaptación, es decir, introducir variaciones en las edades en que aparecen los consecutivos estadios lógicos. Para estas variaciones son de carácter intracultural tanto como intercultural. En el componente intracultural, el factor biopsicológico del desarrollo intelectual encontramos pruebas del valor universal de los procesos cognitivos básicos como la percepción, por ejemplo Cole y Scribner (1977: 33-34) citan: 
El primer estudio comparativo de importancia, acerca de las capacidades cognoscitivas, fue realizado en forma conjunta por antropólogos y psicólogos entre los habitantes del Estrecho de Torres (entre Nueva Guinea y Australia), justamente a fines del siglo XIX; W.H.R. Rivers y sus colegas ejecutaron una extensa serie de tests sobre la función perceptiva, concluyendo que la agudeza perceptiva no difiere notablemente entre el "salvaje" y el europeo normal. Sin embargo, en las funciones cognitivas más complejas como el manejo de silogismos verbales y la formación de conceptos, el factor intracultural evoluciona como consecuencia de los cambios socio-económicos y la labor educativa. Esta hipótesis es sustentada por Vigotsky y Luria, (Cole y Scribner, 1977, 32) por lo que el interés posterior se ha desplazado hacia problemas más complejos que giran en torno a la influencia que las experiencias culturales y ambientales tienen sobre la percepción.

Si bien muchos grupos culturales "primitivos" (vocablo infelizmente empleado) no reconocen en las formas bidimensionales la representación tridimensional, sobre todo para representar la distancia, lo realizan del mismo modo que los niños occidentales entre 6-7 años, estas son observaciones que abundan en favor de la experiencia en los dibujos como medio de representar la tridimensionalidad confirman la existencia de convenciones culturales y que los sujetos van aprendiendo a manejar para compensar los efectos ilusorios de los objetos, y organizar la información de los estímulos que los componen, en una representación de carácter imaginado.

La construcción de la imagen mental trasciende los fenómenos perceptivos y constituye la herramienta intelectiva de los procesos de abstracción: extracción de información sobre las transformaciones de los atributos físicos aparentes o de carácter perceptual en invariantes funcionales que implican la generalización de esquemas de asimilación de los índices perceptivos a imágenes representativas.

Para explicar aprendizajes en los cuales no hay estímulos evidentes perceptualmente y que nos revelan la capacidad de anticipación frente a los objetos como consecuencia de la imagen mental es decir de la capacidad de planificación para responder a posibilidades de movimiento de un objeto, de transformaciones en su figura espacial o temporal; lo cual nos evidencia que la identidad no solo obedece a las leyes de la Gestalt, sino también a haber aprendido respuestas representadas en el esquema corporal y la imagen corporal. Llevar a cabo un entrenamiento equivale, pues a codificar en ellos las pautas culturales de los principios de acomodación.

De ahí que Rivera J.C. (2007) sostiene que el concepto de acomodación y consecuentemente de adaptación del esquema piagetano vincula lo biológico y cultural. En consecuencia, enfatiza que el problema de percepción y cultura, sin reducirse a los modelos de abstractoconcreto, tiene que contemplar el medio ambiente basándose en dos parámetros mínimos: los recursos naturales en sus dimensiones estéticas vs. utilitarias, que en resumen diferencian el encuentro de la Europa monetaria y la América del trueque, determinan las fuentes del poder y el estatus, este último dominante en las sociedades prehispánicas y por tanto a las actividades de una cosmología que ordena la experiencia vital y tecnológica en función de los valores que emanan de ella.

Según Hadson (1901: V), esta razón justifica a que los procesos perceptivos y cultura deben estar presente en toda decisión de intervención en tanto "ninguna investigación de un pueblo estaba completa si no incluía un estudio de su psicología” (Hadson, 1901: V). 


\section{Disonancia cognoscitiva y percepción del valor agregado aplicado a los productos culturales}

Festinger, hacia 1954, plantea la existencia de un estado psicológico de disonancia que afecta al organismo: lo activa y lo dirige, de manera poco confortable, estado a ser reducido aun distorsionando la realidad. Plantea que la disonancia es la inconsistencia entre los elementos afectivos, cognoscitivos o conductuales de una actitud o entre dos elementos cognoscitivos o cualquier otro tipo de inconsistencia cognoscitiva (Muñoz C., 1980: 168). En este caso se trata del valor agregado a productos unos cotidianos y otros olvidados, en alguna medida, entendiéndose por Valor agregado o valor añadido a una característica o servicio extra que se le da a un producto o servicio, con el fin de darle un mayor valor comercial, generalmente se trata de una característica o servicio poco común, o poco usado por los competidores, y que le da al negocio o empresa, cierta diferenciación, comparado con los competidores y entre las diferentes etapas de producción.

Lograr agregar valor requiere de considerar que uno de los fundamentales, sostenidos y beneficiosos factores del valor agregado para los productos elaborados es el de lograr no sólo pensar sino tener "marca". Para Montiel, Eduardo F., es importante tener en cuenta que los esquemas de valor agregado evitan las guerras de precios y si bien los márgenes son menores, lo son con una proyección más clara para el riesgo del capital invertido; las utilidades en ambos casos pueden llegar a ser similares, pero mientras uno mide el pasado, el valor agregado mide el futuro.

Así, se debe realizar un esfuerzo organizado para transformar un producto, para hacerlo más apreciado en un mercado determinado, y se da debido a que tanto el consumidor como el productor buscan mutuamente diferenciarse o simplemente acceder a nuevas prestaciones; de este modo, esa distinción marca muchas veces la preferencia o la satisfacción que se quiere tener o dar se torna complejo, que usualmente se dice de manera popular según el ojo con que se quiera ver, de ahí que "Hay que tener en cuenta un sinnúmero de factores para usar bien el elemento valor agregado. En conclusión, el problema de producir con más o menos valor agregado o costo, no es del consumidor, sino de quien lo produce. La gente exige calidad, frescura, color o pigmentación, peso y sobre todo precio. Pero el éxito depende de la mirada del consumidor. Situación que obliga a pensar en la situación del productor en las zonas rurales, a lo que se añade una autopercepción de las condiciones de trabajo desfavorables.

Con estas consideraciones se estuvo en condiciones de asumir que estimular con miras a poner en valor productos tradicionales, no vistos como productos comercializables y con exigencias de trabajo sostenido bajo una gestión de calidad sostenida compartida no eran necesariamente provocadores de alegría y de abierto optimismo.

\section{Hipótesis}

Los talleres sobre valor agregado afectan significativamente la percepción de los productos culturales en adultos de los caseríos de la comunidad Cruz de Mayo. 


\section{Objetivo}

Identificar en qué medida los talleres sobre valor agregado a los productos culturales afectan la percepción de los productos culturales para su comercialización en los pobladores de los caseríos de la comunidad Cruz de Mayo.

\section{Impacto}

El conocimiento de la percepción económica a expresiones culturales y a su valor agregado es un indicador válido para formular estrategias de gestión de las comunidades campesinas altoandinas según la oferta de productos culturales que favorezcan la inclusion económica; en tanto posibilita:

1. Entender las barreras culturales que impiden los comportamientos proactivos de sus actores para ganar mercado en el contexto de "Sierra exportadora" y la oportunidad de ampliación de mercados con las firmas de los TLC.

2. Idenficar e implementar una línea de investigación de la psicología económica e intervenir en proyectos dirigidos a mejorar los estándares de vida de las poblaciones excluidas socioeconomicamente y proporcionar criteriores para una mejor percepción hacia nuevo sector económico de nuestro país y su implementación; en tal sentido, como toda intervención académica, trae consigo aspectos como:

a. Informar al gobierno local de la provincia de Huaylas, capital Caraz, Ancash, con la firma de un convenio específico a fin de ampliar su cobertura e involucrarlo para participar en proyectos de la formación de una unidad productiva rural.

b. Promocionar la importancia cultural para la identidad y con un aporte significativo en la diversificación del portafolio de empleo para la comunidad campesina Cruz de Mayo.

c. Participación activa de los profesores del I.E.N86515 del caserío Queral, quienes incluirán 4 horas pedagógicas en su plan de estudios para la formación en la recuperación e identificación de productos culturales en una población de 88 niños del nivel primario.

\section{MÉTODO}

Metodología: investigación aplicativa con metodología mixta; Diseño de investigación mixta cualitativa y cuantitativa, con una estrategia de transformación concurrente. 


\section{Población y muestra}

Tabla N. ${ }^{\circ}$ 1. Población del estudio.

\begin{tabular}{|c|c|c|c|c|c|c|c|c|c|c|}
\hline \multirow{3}{*}{$\begin{array}{c}\text { Caserío } \\
\text { Pampacocha }\end{array}$} & \multirow{3}{*}{$\begin{array}{c}\text { Sujetos } \\
509\end{array}$} & \multirow{3}{*}{$\begin{array}{c}\text { Familias } \\
85\end{array}$} & \multirow{3}{*}{$\begin{array}{c}\begin{array}{c}\text { Mayores de } \\
\text { 16 años }\end{array} \\
254\end{array}$} & \multicolumn{3}{|c|}{ Muestra e $=0,05$} & \multicolumn{3}{|c|}{$\begin{array}{c}\text { Muestra e }=\mathbf{0 , 1} \\
\text { Teórica }\end{array}$} & \multirow{3}{*}{$\begin{array}{c}\text { Muestra e }=\mathbf{0 , 1} \\
\text { Real } \\
42\end{array}$} \\
\hline & & & & \multirow{2}{*}{103} & 51 & M & \multirow{2}{*}{38} & 19 & M & \\
\hline & & & & & 52 & V & & 19 & V & \\
\hline \multirow{2}{*}{ Queral } & \multirow{2}{*}{241} & \multirow{2}{*}{50} & \multirow{2}{*}{125} & \multirow{2}{*}{52} & 28 & M & \multirow{2}{*}{19} & 10 & M & \multirow{2}{*}{34} \\
\hline & & & & & 24 & V & & 9 & V & \\
\hline \multirow{2}{*}{ Miramar } & \multirow{2}{*}{208} & \multirow{2}{*}{43} & \multirow{2}{*}{106} & \multirow{2}{*}{44} & 23 & M & \multirow{2}{*}{16} & 8 & M & \multirow{2}{*}{6} \\
\hline & & & & & 21 & $\mathrm{~V}$ & & 8 & $\mathrm{~V}$ & \\
\hline \multirow{2}{*}{ Chinchay } & \multirow{2}{*}{117} & \multirow{2}{*}{16} & \multirow{2}{*}{61} & \multirow{2}{*}{25} & 13 & M & \multirow{2}{*}{9} & 5 & M & \multirow{2}{*}{16} \\
\hline & & & & & 12 & V & & 4 & V & \\
\hline \multirow{2}{*}{ Totales } & \multirow{2}{*}{1075} & \multirow{2}{*}{194} & \multirow{2}{*}{546} & \multirow{2}{*}{224} & 116 & M & \multirow{2}{*}{82} & 42 & M & \multirow{2}{*}{98} \\
\hline & & & & & 108 & V & & 40 & V & \\
\hline
\end{tabular}

Fuente: Posta de Salud de Pampacocha (Paredes, M y De Miguel, J., 2004).

La comunidad de estudio se ubica en la falda de los nevados más altos de la Cordillera Blanca, en la zona occidental de los andes peruanos, sobre los $3000 \mathrm{msnm}$, que vienen influyendo sobre la cultura y la civilización desde la época preinca. La importancia del desarrollo de la vida humana depende de su relación con el medio físico, la población de 1075 pobladores de los Caseríos de Miramar, Queral, Chinchay, Pampacocha del distrito de Caraz, Huaylas, Ancash.

La muestra fue tomada en dos de los cuatro caseríos, Pampacocha y Queral, el más próspero y el más pobre, respectivamente, con una muestra inicial de 92 participantes y una final de 23.

Tabla N. ${ }^{\circ}$ 2. Distribución muestral de la comunidad Cruz de Mayo por género, estado civil, condición y grado de estudio, del distrito de Caraz, provincia de Huaylas, región Ancash*.

\begin{tabular}{|c|c|c|c|c|c|c|c|c|c|c|c|c|c|}
\hline & & \multicolumn{2}{|c|}{ Chinchay } & \multicolumn{2}{|c|}{ Miramar } & \multicolumn{2}{|c|}{ Pampachocha } & \multicolumn{2}{|c|}{ Queral } & \multicolumn{2}{|c|}{ Subtotal } & \multicolumn{2}{|c|}{ Total } \\
\hline & & Cuenta & $\%$ & Cuenta & $\%$ & Cuenta & $\%$ & Cuenta & $\%$ & Cuenta & $\%$ & Cuenta & $\%$ \\
\hline \multirow{2}{*}{ Género } & Masculino & 12 & 13 & 9 & 10 & 22 & 24 & 44 & 48 & 87 & 95 & & \\
\hline & Femenino & 0 & 0 & 1 & 1 & 0 & 0 & 4 & 4 & 5 & 5 & 92 & 100 \\
\hline \multirow{4}{*}{$\begin{array}{l}\text { Estado } \\
\text { civil }\end{array}$} & Casado & 7 & 8 & 7 & 8 & 13 & 14 & 18 & 20 & 45 & 49 & & \\
\hline & Conviviente & 3 & 3 & 1 & 1 & 9 & 10 & 21 & 23 & 34 & 37 & & \\
\hline & Soltero & 2 & 2 & 1 & 1 & 0 & 0 & 8 & 9 & 11 & 12 & & \\
\hline & Viudo & 0 & 0 & 1 & 1 & 0 & 0 & 1 & 1 & 2 & 2 & 92 & 100 \\
\hline \multirow{3}{*}{ Condición } & Comunero & 10 & 11 & 8 & 9 & 11 & 12 & 15 & 16 & 44 & 48 & & \\
\hline & Hijo de comunero & 2 & 2 & 2 & 2 & 6 & 7 & 17 & 18 & 27 & 29 & & \\
\hline & Propietario & 0 & 0 & 0 & 0 & 5 & 5 & 16 & 17 & 21 & 23 & 92 & 100 \\
\hline \multirow{4}{*}{$\begin{array}{l}\text { Grado de } \\
\text { estudio }\end{array}$} & Iletrado & 0 & 0 & 0 & 0 & 2 & 2 & 1 & 1 & 3 & 3 & & \\
\hline & Prim. completa & 2 & 2 & 1 & 1 & 6 & 7 & 10 & 11 & 19 & 21 & & \\
\hline & Prim. incompleta & 10 & 11 & 8 & 9 & 14 & 15 & 35 & 38 & 67 & 73 & & \\
\hline & Sec. completa & 0 & 0 & 1 & 1 & 0 & 0 & 2 & 2 & 3 & 3 & 92 & 100 \\
\hline
\end{tabular}

\footnotetext{
* Datos directos del Registro de Participantes.
} 


\section{Para el diagnóstico}

La identificación de la percepción del conocimiento de las expresiones culturales en productos de bienes y servicios se realizó de la siguiente manera:

a. Coordinación y aceptación de la comunidad, designando a un miembro como representante ante el presente estudio, con la firma de un Acuerdo.

b. Trabajo de campo de exploración; para lo cual en un inicio se realizó mediante la técnica denominada "El vagabundeo" y ocasionalmente una conversación con intérprete, con aquellos participantes que tiene mayor dominio del castellano.

c. Aplicación de la lista de cotejo, en base al listado de productos culturales de la UNESCO.

d. Identificación de productos culturales con el potencial para la generación de valor agregado, sustentado en los mediadores psicosociales de la conducta propios de su contexto, y a ser gerenciados por sus mismos actores descritos según la representación social de su organización de su comunidad.

e. Se identificó y coordinó con cada participante representante de cada familia, unidad de observación.

f. Se desarrolló el proceso de estimulación en los participantes de los productos culturales con valor agregado para su comercialización incluyendo las evaluaciones de entrada y de salida.

\section{Para la aplicación del método cuasiexperimental}

En la parte cuantitativa con el método cuasiexperimental pre y pos test; donde las variables independiente (VI), Taller sobre el valor agregado a los productos culturales, la variable dependiente (VD), La percepción al valor agregado de los productos y bienes, y la variable de control la zona geográfica de los cuatro caseríos; se prosiguió los siguientes pasos:

a. El proceso de aplicación de la Escala de percepción para evaluación antes (pre test) y déspues (pos test) de VD: percepción del conocimiento de los productos culturales:Bienes y servicios.

b. Intervención: La VI; Taller de información de valores agregados de bienes y servicios de las expresiones culturales identificadas con la escala de cotejo (Ver Anexo)

c. Evaluación de salida (post test) de VD: percepción del conocimiento de los productos culturales: Bienes y servicios.

\section{Diseño de técnicas e instrumentos}

La recolección de datos se hizo mediante:

a. Entrevistas cualitativas y la técnica "El vagabundeo".

b. Focus group.

c. Se confeccionó las Escalas de Percepción (E.E.P.C.- 2007), bajo la técnica de Likert, con 18 ítemes y cuatro grados de valoración, con una confiabilidad igual a 0,93. 
d. Lista de cotejo, según especificaciones del listado de la UNESCO.

e. Estímulo visual de la VI: Banner: Taller sobre el valor agregado a los productos culturales.

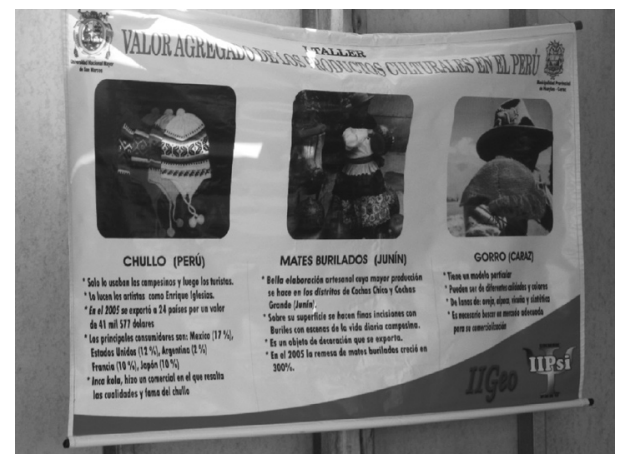

Diseño ad hoc para el Taller, VI: Yanet Montoro. Colaboradora del Equipo de Investigaciòn.

\section{Técnicas de Análisis estadísticos}

Para comparar la percepción y conocimiento del valor agregado a productos culturales entre la comunidad se aplicaron estadísticos descriptivos, prueba de normalidad y el estadístico t de student para grupos apareados.

\section{RESULTADOS}

Mediante la lista de cotejo, observación participante y entrevista, se obtuvo los siguientes resultados en la zona de investigación, cuya belleza natural está siendo puesta en valor para actividades de turismo de aventura y vivencial. Como muestran la montaña y el nevado Santa Cruz, tutelares de la comunidad campesina Cruz de Mayo.

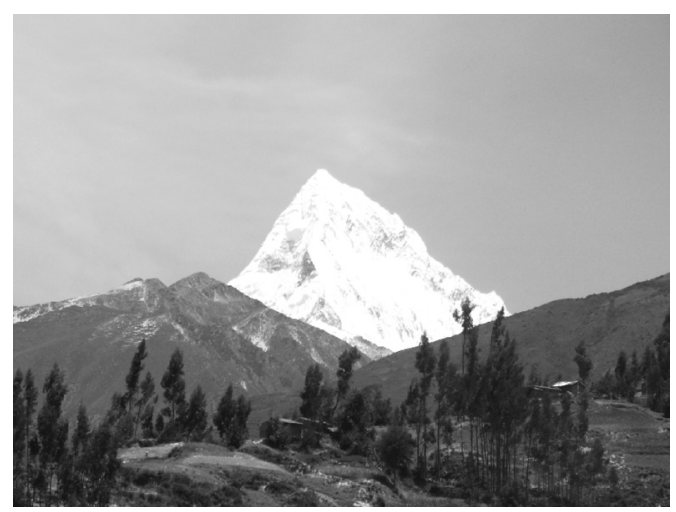

Vista del nevado Santa Cruz desde la laguna Miramar. Foto: Equipo de Investigación. 


\section{Bienes culturales identificados}

\section{Textiles}

a. Gorro infantil; b.Chalinas; c. Tejidos a telar: Huatos, alforjas, frazadas, costales caracterizado en forma y matices e intercalado en colores crema y marrón preferentemente. Los hay también multicolores según pedido. También hemos visto la utilización de fibras sintéticas, con palitos y croché.

1. Diseño de gorro para niño: Este gorrito lo tejen o compran para los bebés de la comunidad. Su diseño es particular, la parte superior cubre como un pequeño casquete o semihemisferio, y alrededor cuelgan como gorgueras o tejidos ondulantes protegiendo los oídos y la nuca del infante y en la parte delantera se proyecta como una visera. El diseño parece mezclar elementos de origen virreinal con republicanos, que aún faltan precisar. Diseño ubicado inicialmente en el caserío de Miramar.

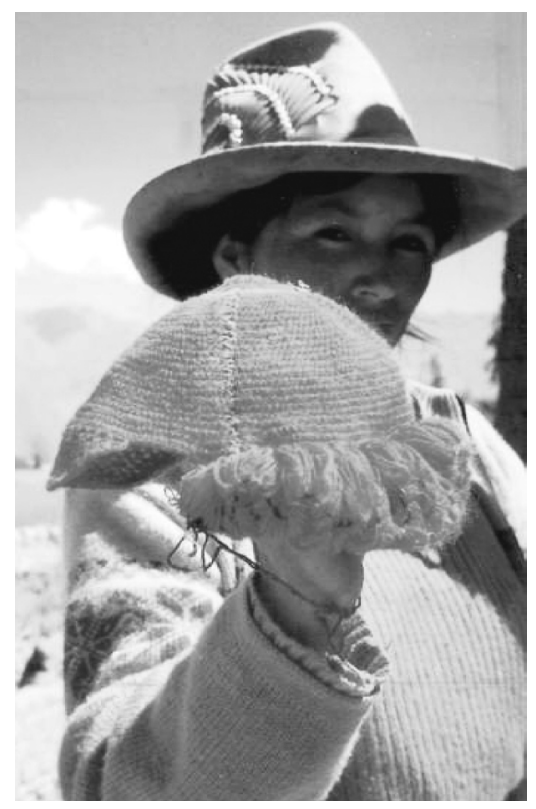

Gorro infantil típico de Huaylas.

Foto. Equipo de Investigación.

2. Yakuma o jakuma: especie de chompa con mangas, espalda triangular y una tapita adelante. Tejido con lana, color blanco y negro, rojo y blanco, amarillo y granate, formando figuras geométricas. Prenda ancestral del caracino que aún perdura (Malca, Landavery, 1993: 102). Su uso frecuente es porque se dedican a la extracción, o especialmente para cargar hielo de los nevados, requerido para la elaboración de helados y raspadilla, muy propio y apetecible a lo largo del Callejón de Huaylas. 
3. Frazadas, costales, alforjas con diseños geométricos.

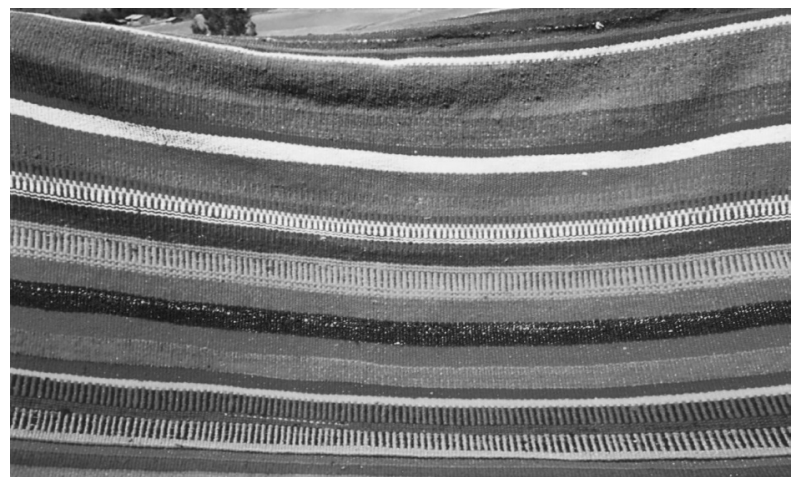

Diagrama con líneas clásicas de los tejidos a telar. Foto. Equipo de Investigación.

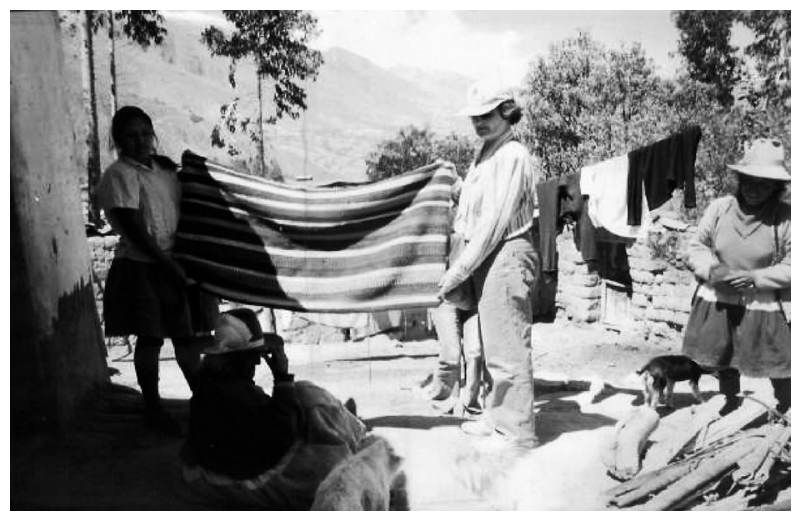

Frazada de lana de oveja, en el domicilio del fabricante. Foto. Equipo de Investigación.

\section{Expresiones culturales de las actividades desarrolladas en sus respectivos procesos productivos agrícolas y ganaderos}

Son las actividades socioeconómicas-culturales que están vinculadas al calendario agrícola; se inician aquellas fiestas propias de la zona de estudio, identificadas por sus artifíces como naturales, rutinarias "siempre lo hacemos así, "es nuestra costumbre" refiriéndose a los procesos de siembra y cosecha: tanto de papa; cebada; arveja; quinua; quiwicha, olluco, entre otros productos de su dieta, los mismos que pueden ser desarrollados para el turismo vivencial rural como paquetes programados para el gusto del consumidor. Son fiestas donde expresan no sólo su tecnología, sino la manera de hacer las cosas con su afectividad, alegría y culto en su ejecución; actividades que transmiten peculiaridades a ser admiradas, sustentantadas en la "productividad" como una dimensión psicosocial significativa en la representación social de la construcción de su organización de su comunidad (Paredes, M. y De Miguel, J. Ma., 2004). 
1. Siembra y cosecha de la papa.

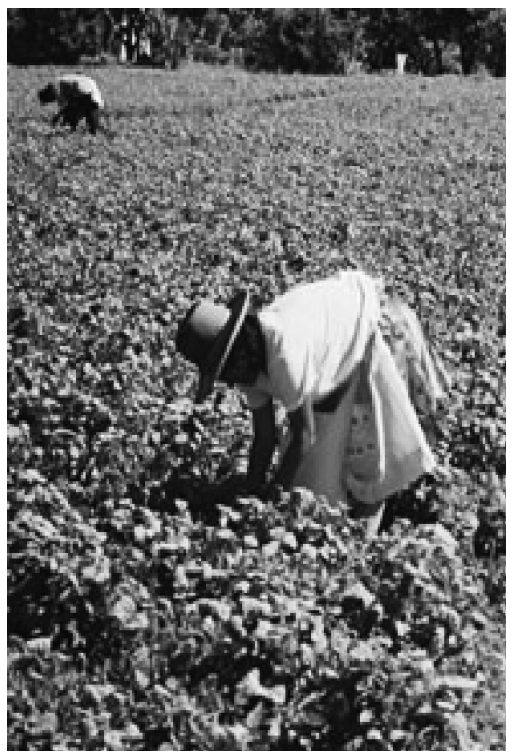

Siembra de la papa: no hay distinción de género. Foto: Equipo de investigación.

2. Trilla de cereales: trigo, cebada, Labranza; mediante el arado.

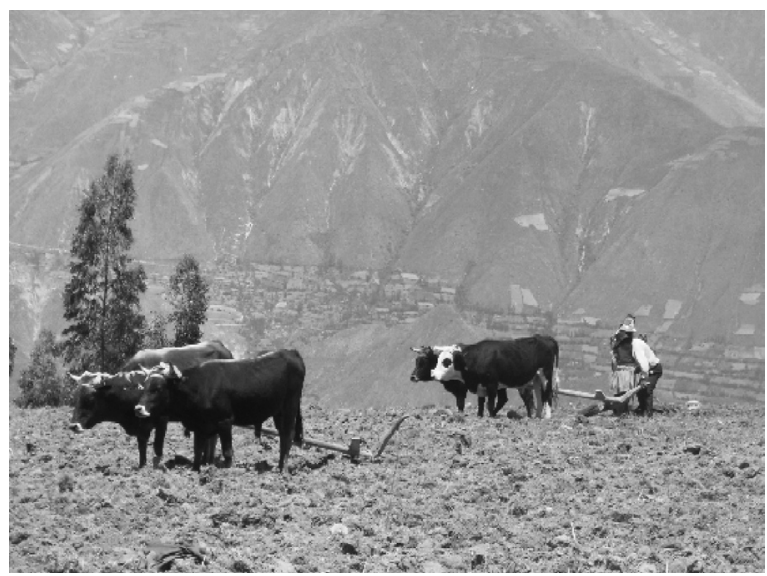

Proceso de labranza con bueyes sin distinción de género en el Caserío de Miramar. Fotografia: Equipo de investigación.

3. Fiestas culturales: fiestas y conmemoraciones patronales: música; danza, historias, cuentos, poesía. 


\section{Técnicas de los telares}

Respecto a la tradición textil sobre Huaylas y en particular Caraz, es importante notar que se producen mantas o frazadas, ponchos y sacos de bayeta. La costumbre de algunos miembros de la comunidad de Queral y Miramar varía según su acercamiento a la ciudad. Por ejemplo, hemos visto trabajar a algunos hombres el telar de cintura atado a un árbol. En ese caso se utiliza lana de oveja, de dos tonos, uno crema y otro marrón. Las mujeres todavía tienen la costumbre de hilar, colocan la lana de oveja en un palo y en otro palo llamado Huso, que tiene un objeto con peso (papa seca, cerámica o piedra), giran estirando la fibra hasta convertirla en un hilo largo y liso de grosor diverso.

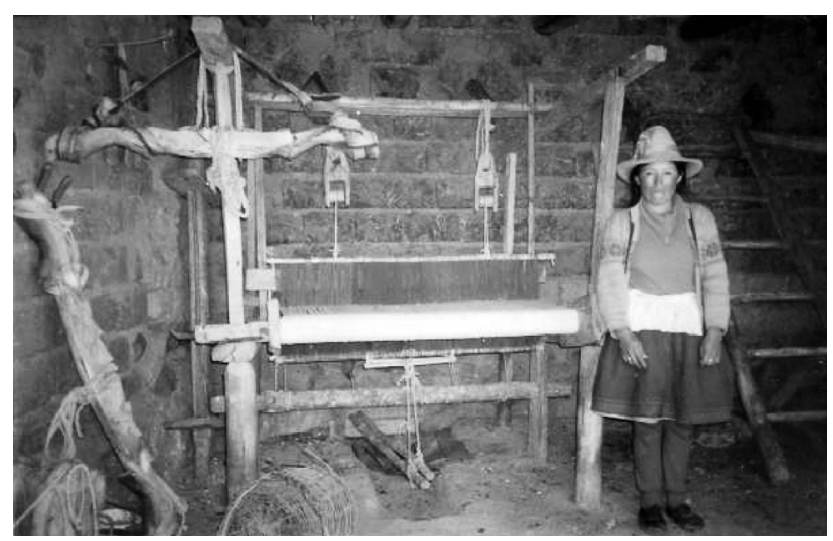

Telar: Caserío de Miramar.

Foto: Equipo de investigación.

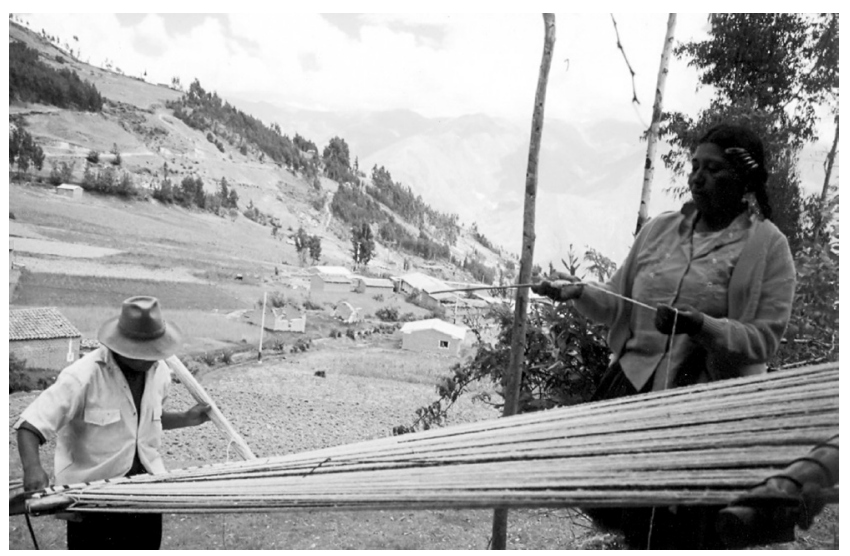

Telar de cintura en el Caserío de Queral.

Foto: Equipo de investigación. 


\section{Confiabilidad del instrumento}

El instrumento de medición denominado "Escala de Expresiones en Productos Culturales - Caraz 2007" (E.E.P.C. 2007) formado por 20 reactivos presenta una confiabilidad de 0.90, calculado mediante el método de consistencia interna, con la fórmula KR20.

Validez del instrumento

La validez dada al instrumento es de contenido y fue calculada mediante la determinación de la capacidad de discriminación entre el grupo superior y el grupo inferior mediante la prueba $\mathrm{t}$.

Tabla N. ${ }^{\circ}$ 3. Validez de contenido de la "Escala de Expresiones Culturales en Productos - Caraz 2007” (E.E.C.P. 2007)

\begin{tabular}{ccccccccccccccccccccc}
\hline & 1 & 2 & 3 & 4 & 5 & 6 & 7 & 8 & 9 & 10 & 11 & 12 & 13 & 14 & 15 & 16 & 17 & 18 & 19 & 20 \\
& P1 & P2 & P3 & P5 & P7 & P10 & P11 & P13 & P14 & P16 & P17 & P18 & P19 & P20 & P22 & P23 & P24 & P25 & P26 & P27 \\
$\mathrm{t}$ & 4.3 & 2.1 & 3.8 & 5.3 & 4 & 4.8 & 3 & 2.1 & 4 & 5.9 & 5.5 & 4.8 & 5.3 & 5.8 & 3.5 & 3.6 & 5 & 4.7 & 3.6 & 3.7 \\
\hline
\end{tabular}

Todos los ítemes presentados discriminan entre grupo superior y grupo inferior, constituyendo una medida válida de la percepción de expresiones culturales en la población objetivo.

Tabla N. ${ }^{\circ}$ 4. Estadísticos descriptivos de los resultados de la prueba de entrada y de salida.

\begin{tabular}{|c|c|c|c|}
\hline \multirow{2}{*}{\multicolumn{2}{|c|}{$\mathrm{N} \quad$ Valid }} & \multirow{2}{*}{$\frac{\text { SALIDA }}{23}$} & \multirow{2}{*}{$\begin{array}{r}\text { ENTRADA } \\
23\end{array}$} \\
\hline & & & \\
\hline Media & & 40,61 & 42,87 \\
\hline Mediana & & 41,00 & 44,00 \\
\hline Moda & & $40^{\mathrm{a}}$ & 45 \\
\hline Desviación & & 9,834 & 7,659 \\
\hline Varianza & & 96,704 & 58,664 \\
\hline Sesgo & &,- 014 &,- 265 \\
\hline Error estánd & de Sesgo & ,481 & ,481 \\
\hline Kurtosis & &,- 284 &,- 781 \\
\hline Error estánd & de Kurtosis & ,935 & ,935 \\
\hline Rango & & 37 & 27 \\
\hline Suma & & 934 & 986 \\
\hline \multirow{3}{*}{ Percentiles } & 25 & 33,00 & 35,00 \\
\hline & 50 & 41,00 & 44,00 \\
\hline & 75 & 48,00 & 49,00 \\
\hline
\end{tabular}

a. Existen múltiples modas. Se muestra el valor más pequeño. 


\section{Contraste de hipótesis}

La pregunta motivo de la presente investigación se refiere a la posibilidad de modificar la percepción hacia las expresiones culturales en la población adulta de la comunidad Cruz de Mayo. Para ello se ha usado un diseño antes-después.

Tabla N. ${ }^{\circ}$ 5: Evaluación antes y después de la percepción de la variable independiente: Valor agregado a los productos culturales

\begin{tabular}{cccccc}
\hline Media & $\begin{array}{c}\text { Desviación } \\
\text { estándar }\end{array}$ & $\begin{array}{c}\text { Error de } \\
\text { medición }\end{array}$ & $\mathrm{t}$ & $\mathrm{G} .1$ & $\begin{array}{c}\text { Significación } \\
\text { Para } \alpha=.05\end{array}$ \\
\hline$-2,261$ & 5,092 & 1,062 & $-2,129$ & 22 &, 045 \\
\hline
\end{tabular}

* = Significativo al 0.05

\section{RESULTADOS}

Se encuentra diferencia significativa $t=-2.19$ (Tabla N. ${ }^{\circ}$ 5) a favor de la percepción de la aplicación de valor agregado a los productos culturales de la primera evaluación, al contrastarse los datos luego de la intervención con el VI Taller de información sobre los valores agregados de bienes y servicios de las expresiones culturales, en productos culturales comerciables; asimismo se observar que la percepción en la segunda evaluación ha disminuido pese a seguir siendo positiva.

Este resultado, que a pesar de la diferencia de la primera con la segunda evaluación, y con un resultado positivo de percepción en ambas mediciones, permite aseverar que se ha generado una disonancia cognitiva en la población estudiada, la cual no se hubiera producido sin la acción del VI Taller de Información, por cuanto la percepción del producto cultural es susceptible a ser afectada cuando se le otorga propiedades de un bien comercial, porque entran en conflicto dos elementos cognoscitivos: la resistencia a comercializar con algo tradicional de su cultura y el escepticismo de que tales productos pudieran tener un valor comercial capaz de convertirse en un negocio rentable, a lo cual podríamos añadir el poco conocimiento que los más jóvenes tienen de la existencia de los productos tradicionales, los cuales son una alternativa a su crecimiento económico, es decir, que hay nociones que interfieren con la visión como negocio de estos productos culturales.

Hay otros factores que podrían tener alguna injerencia en la percepción del valor agregado a los productos culturales en sí mismos y también justamente por no contar con información de dichos factores que explique cuantitativamente la disminución en la misma dirección positiva de la percepción en la segunda evaluación, deja abierta las puertas a las siguientes reflexiones:

- La razón por la que atribuimos que hubo disminución significativa se debe a la resistencia al cambio, puesto que los miembros de los caseríos de la comunidad, 
si bien tienen como fortaleza el trabajo comunitario y el intercambio de bienes de subsistencia, como alimentos, enseres, leña, etc., al parecer de ninguna manera están dispuestos a aceptar la distribución de las ganancias provenientes que vienen de aquellos productos comerciables como la arveja.

- La efectividad de la información del taller se da por la presencia del conocimiento sobre el valor agregado a pesar de la disonancia cognitiva o la disminución en la direccionalidad de la actitud.

- A este factor le agregamos la barrera del bilingüismo que dificulta la comunicación semántica, repercutiendo en la decodificación adecuada de los conceptos.

\section{DISCUSIÓN}

Se ha identificado y clasificado en tres grandes rubros a partir del registro preliminar de las expresiones culturales para ser productos culturales comerciales, los mismos requieren perfiles para su formulación de proyectos de desarrollo de cada producto, en las actividades de producción, mercado y gestión; con sus respectivos planes de capacitación y adiestramiento. A continuación se señala a ser desarrollados en productos culturales comerciables según los lineamientos de la UNESCO:

1. Productos Textiles: Según su tecnología propia con desarrollo de cada producto de su expresión cultural en producto comerciable, entre ellos podemos destacar el "gorrito infantil”, el que debe investigarse como producto típico del Callejón de Huaylas y aquellos telares nombrados: frazadas, alforjas, huatos, entre otros.

2. Productos de fiestas tradicionales según el calendario agrícola, de sus productos: de siembra y cosecha: papa, olluco, mashua; arveja; quinua; quiwicha; trilla del trigo, cebada, centeno; empleo de la flora silvestre, preferentemente que a pesar que son concebidas como tareas de sus actividades propias y rutinarias pueden ser desarrolladas como productos para el turismo rural vivencial, a formularse y desarrollarse en paquetes turísticos debidamente estructurados y planificados.

3. Fiestas culturales: fiestas y conmemoraciones patronales: música; danza, historias, cuentos y poesía.

\section{CONCLUSIONES}

1. Escala de Expresiones Culturales en Productos - Caraz 2007 (E.E.C.P. 2007). La E.E.P.C. 2007 presenta validez de contenido, pues sus ítems discriminan entre el grupo superior y el grupo inferior.

2. Escala de Expresiones Culturales en Productos - Caraz 2007 (E.E.C.P. 2007) es confiable, por el método de consistencia interna.

3. Se rechaza la hipótesis nula, Ho, de ahí se reafirma que los talleres sobre valor agregado afectan significativamente la percepción de los productos culturales en jóvenes y adultos de los caseríos de la Comunidad Campesina Cruz de Mayo. 
4. Se identifica expresiones culturales a ser desarrollados en productos culturales comerciables, Textiles como por ejemplo, el "Gorrito Infantil de Huaylas", telares a la cintura; actividades según el calendario agrícola, como las fiestas de cosecha y siembra para paquetes turísticos rurales vivenciales.

\section{RECOMENDACIONES}

1. Incorporar a la mecánica de talleres otros procedimientos, sea porque la intensidad inicial de la actitud hacia al valor agregado del producto cultural disminuye al parecer por efecto de la información o porque se encuentra resistencia al cambio.

2. Afrontar las barreras planteadas por el bilingüismo mediante agentes que faciliten que el mensaje sea transmitido con mayor nitidez en ambas direcciones.

3. Explorar los aspectos históricos y antropológicos para encontrar elementos que reviertan la percepción negativa hacia la constitución de negocios comunes.

4. Plantear a la Municipalidad de la Provincia de Huaylas, que apoye la investigación y el Instituto Nacional de Cultural del "Gorrito Infantil" si lo amerita como expresión cultural propia de la zona.

\section{NOTA DE RECONOCIMIENTO}

Dejamos expreso nuestro reconocimiento al Consejo Superior de Investigaciones de la UNMSM, IIPsi de la Facultad de Psicología, por su financiamiento; a la Municipalidad Provincial de Huaylas, Caraz por su apoyo, y nuestra gratitud a los participantes, miembros de los caseríos: Pampacocha, Queral, Miramar y Chinchay de la comunidad campesina Cruz de Mayo, que sin su acogida hubiera sido imposible llevar cabo el presente estudio. Asimismo al Ing. Laureano Valentín, Soc. N. Dora Valencia P., Bch. Alejandra Torres, Codra. Yanet Montero y al comunero Sr. Camilo Alejo Leiva por su colaboración en el estudio.

\section{REFERENCIAS BIBLIOGRÁFICAS}

1. Adams, N. y Valdivia N. (1994). Los otros empresarios. Ética de migrantes y formación de empresas en Lima. Lima: IEP.

2. Cartwright D. y Zander, A. (1985). Dinámica de grupos, investigación y teoría. México: Trillas.

3. Cole, M. y S. Scribbler (1977). Cultura y pensamiento. Relación de los procesos cognoscitivos con la cultura. México: Limusa.

4. Cole, M. (1999). Psicología Cultural. Madrid: Morata.

5. Descouvières, C. (1998). Psicología económica, Temas escogidos, Chile: Editorial Universitaria. 
6. Festinger, Leo, Stanley, Schachter (1985). Funcionamiento de los estándares de grupo. En Cartwright y Sanders. Dinámica de Grupos, Investigación y Teoría. México: Trillas.

7. González, R. (1989). Psicología del aprendizaje. Lima: Biblioteca de Psicología Peruana.

8. Hudson, T. (1983). Correspondences and numerical differences between disjoint sets. Child Development, 54. 84-90.

9. IIGEO Plan de Desarrollo de la Comunidad Campesina Espiritu Santo de Chacayán 2006-2016. UNMSM / CENTROMINPERÚ. Lima-Perú IIGEI.

10. Kapsoli W. (1989). Historia y psicología del indio. Lima: Editora Caribe S.A.

11. Katona G. (1965). Análisis psicológico del comportamiento económico. Madrid: RIALP.

12. Malca Landavery, J.G. (1993). Ancash y su policroma indumentaria.

13. Muñoz C. Carlos y colaboradores (1980). Formación y cambio de actitudes en psicología social. México: Editorial Trillas.

14. Paredes Tarazona, M.; De Miguel J.M. (2004). Construcción social de la comunidad para el desarrollo local del Callejón de Huaylas, Caraz. Revista de Investigaciones en Psicología. Lima: UNMSM/ FPs/ IIPs

15. Paredes T., M.; De Miguel C., JMa.; Hernández V., H.; Vicuña P., L.; et al, (2005). El papel de la construcción social de la organización con respecto a la percepción de las condiciones de trabajo y el manejo del medio ambiente en cuatro caseríos de una comunidad campesina andina: Caraz, Ancash, en Revista del Instituto de Investigación, Facultad de Ingeniería Geológica, Minera, Metalúrgica y Geográfica, UNMSM, Vol. $8 \mathrm{~N}^{\circ} 16,57-67$.

16. Paredes Tarazona, Mildred; Hernández V. Héctor M.; Vicuña Peri, Luis A.; De Miguel Calvo, Jesús María; Aliaga, Roberto; Laguna, Miriam (2007). Percepción del conocimiento de la demanda de productos y percepción hacia la interdependencia económica en el Caserío Queral, Caraz, Ancash. Revista de Investigaciones en Psicología, Lima, Junio de 2007, Vol 10 N²1, UNMSM/ FPs/ IIPs.

17. Quintanilla, Ismael, (2003), un premio Nobel para nuevas perspectivas en la investigación económica y psicológica papeles del psicólogo, enero-abril, año/vol. 23, número 084 Consejo General de Colegios Oficiales de Psicólogos, España, Madrid, España pp. 83-92.

18. Santoro, Eduardo y colaboradores (1980). "Percepción social". En Psicología Social México: Editorial Trillas.

19. Solís Fonseca, Gustavo (2006). Diversidad e interculturalidad: reto y posibilidad en la universidad. Discurso de orden. Inaguración del Año Académico 2006. UNMSM, Rectorado, Lima, Marzo 2006 
20. UNESCO, (2005). Adopción de una convención sobre la diversidad cultural UNESCO. Mono/05/384-es Http:/europa.eu.int/comm/culture/portal/action/diversit/ unesco-en.htm

21. UNESCO (2004). Lista exhaustiva de bienes y servicios culturales, anteproyecto de convención de la UNESCO sobre la protección de la diversidad cultural de los contenidos culturales y las expresiones artísticas en: www.octaedro.org.ec

22. Uribe, Patricia (2005). A propósito de las negociaciones del TLC; cultura-comercio: ¿se excluyen? El Comercio a4 Opinión. 21 de noviembre de 2005.

23. Witkin, H y Goodenough, D. (1981), Cognitive styles-essence and orgins: Field dependence and field independence. New York: International Universities. 